\title{
Mini Access Spine for Fixation of Thoracolumbar Spine Fractures
}

\author{
Bahaa Ali Kornah*, Hesham Mohamed Safwat, Mohamed Abdelaziz, Mohamed Ibrahim Abuelesoud, \\ Tharwat Abdel Ghany, Nagy Saleem and Mohamed Abdel AAl \\ Department of Orthopedic Surgeon, Egypt
}

*Corresponding author : Bahaa Ali kornah, Department of Orthopedic Surgeon, Al-

Received Date: February 09, 2019

Azhar University, Cairo, Egypt.

Published Date: March 14, 2019

\begin{abstract}
Introduction: The aim of treatment of spinal fractures is to resorted stability. Spine fractures are serious injuries can lead to neurological complications. The surgical technique can be either through posterior midline incision or paraspinal posterior open approaches or Percutaneous. The goal of this study is to evaluate the effectiveness of Mini access approach for treatment of dorsolumbar fractures by mini open surgical using ordinary pedicle screw fixation. This study involves Patients of acute traumatic single level dorso-lumbar spine fractures requiring surgical intervention. Thirty two (22 male, 10 female), age range 17-52 years (mean $31.1 \pm 7.9$ yrs) with dorso-lumbar fractures (D12:8, L1:17, L2:4, L3:3) with TLICS score>6 were studied (Feb 2009-Feb 18). Total of 128 screws were used of which 3 screws were mal positioned (2.3\%). Open conversion was done in two cases (6.25\%) due to difficulty in screw positioning.
\end{abstract}

Result: No patient had post-operative neurological deterioration. Operative time (minutes) 65.0 \pm 23.2 . The average blood loss is $94 \mathrm{ml}$ Fluoroscopy time $5.2 \pm 2$ (seconds). Postoperative hospital stay (days) $3.1 \pm 1.8$ days. The follow-up period an average of 10 months. The Accuracy rate of screws placement Type I: position acceptable in 108 screws. In 16 screws were classified as Type II: placement unacceptable. In 3 screws were classified as Type III: screws that are causing neurovascular injury) all cases causing radicular pain).

Conclusion: We conclude that Mini access Spine surgery for pedicle screws fixation is a safe, reliable, cost effective technique with favorable results in acute polytrauma cases requiring standalone ligamentotaxis. It is cost effective, minimal soft tissue dissection with short hospital stay. The authors believe that the data support its use and that this technique may be applicable for cases not needs spinal decompression, Mini access spinal technologies enable surgeons to achieve the same surgical objectives as with a traditional, open procedure.

Keywords: Spine fixation; Minimal spine fixation; Pedicular screw fixation; Fr. Spine; Mini access spine exposure; Mini-open procedures

\section{Introduction}

The aim of treatment of spinal fractures is to resorted stability. Spine fractures are serious injuries can lead to neurological complications. It is widely accepted that thoracolumbar burst fractures should be addressed surgically [1].

The pedicular screw fixation becomes a golden stander to treat this condition with or without decompression. Pedicle screw systems engage all three columns of the spine and can resist motion in all planes. Several studies suggest that pedicle screw fixation is a safe and effective treatment for many spinal disorders $[2,3]$. Short-segment pedicle fixation (SS) is a popular option. Dick et al. [4] have developed the SS stabilization for the operative treatment of thoracolumbar and lumbar fractures. However, there is a controversy as far as the results of this instrumentation are concerned. The choice of treatment option in the absence of a neurological deficit depends on the Thoracolumbar Injury Classification and Severity Score (TLICS) scores [4,5].

This classification not only highlights the fact that axial load transmission travels through the vertebral bodies and Intervertebral discs, it is also a scoring points system that can be used preoperatively to: Predict screw breakage when short 
segment, posteriorly placed pedicle screw implants are being used and Select fractures needing further anterior reconstruction. Those fractures with a combined score of 6 or less are suitable for shortsegment posterior spinal instrumentation, while those with a 7 or higher require either long-segment fixation or circumferential fusion. For insertion of pedicular screws by Standard techniques for pedicle screw fixation which involve open exposures and extensive muscle dissection $[5,6]$.

Surgical technique can be either through posterior midline incision or paraspinal posterior open approaches or Percutaneous. In this paper we will highlighted on mini access approach mini access exposure (mini-open procedures) to insert the pedicular screws and fixation of spine fractures. In this technique the authors used the ordinary pedicular screws. Also, no need to use a modified screws or special screws set with extension sleeve that would allow for remote manipulation of the polyaxial screw heads and remote engagement of the screw locking mechanism and unique rod insertion device. Also, less radiological exposure. The aim of this paper is to describe a mini access exposure (mini-open procedures) to spine for fixation of the thoracolumbar and lumbar spine fractures technique using ordinary pedicular screws and instrumentation.

\section{Patient and Method}

The present prospective study was conducted at El Hussein university Hospital and Bab El Shaaria AL -Azhar University from 2009-2018. This prospective study included 32 patients with Fractures spine between 2009 and 2018. This study included 32 patients with Fractures spine between 2009 and 2018. Fall from height /staircase was the most common mode of injury in 20 patients followed by road traffic accident in 11 and in one case drop of heavy box over the back while working.

There were 22 males and 10 females. Age of patients ranges from 17 to 52 years (mean 31.1 \pm 7.9 yrs). The study included

17 patients with fracture lumbar Spine (L1) and

8 patients with dorsal (D12) fractures and

4 patients with (L2) fractures and

2 patients with (L3) fractures.

All cases underwent FOR mini access SPINE FIXATION using shot segment pedicular screws. The inclusion criteria using Thoracolumbar Injury Classification System (TLICS) [7]. The load sharing classification for burst fractures met these criteria. The study including 32 fractures with a combined score of 6 or less was selected as they are suitable for short-segment posterior spinal instrumentation, also not need for decompression while those with a 7 or higher which need decompression of medullary canal were excluded.

\section{Selection criteria}

All the Patients with TLICS score of 6 or less, without neurological deficit, without needing a decompression of the neural elements with a mechanically unstable burst fracture, reduce displaced bony fragments by ligamentotaxis within 48-72 hours after injury. The follow up period ranged from 6 to 18 months postoperative with an average of 10 months.

\section{Technique}

Preoperative management: The patient was placed on a firm mattress. Catheterization, Parenteral antibiotics were administrated 2 hours before surgery and continued for about 2 days postoperatively.

Operative procedure: Anesthesia was given to the patient while lying supine beside the operating table. Hypotensive anesthesia was used to decrease the blood loss intraoperatively, and to facilitate homeostasis.

Position of patient: The patient is positioned onto a radiolucent table prone on two horizontally placed padded bolsters (one at the level of sternum and another one at the level of anterior iliac spine) or a frame. The abdomen should hang free to avoid increased intraabdominal pressure to prevent excessive bleeding. Adequate padding needs to be provided to elbows and knees to avoid pressure sores. The head is rested either in a horse shoe ring or a Mayfield rest to avoid pressure on the eyes. Hips and knees were moderately flexed to prevent stretching of the nerve roots. Then, sterilization and draping were done (Figure 1).

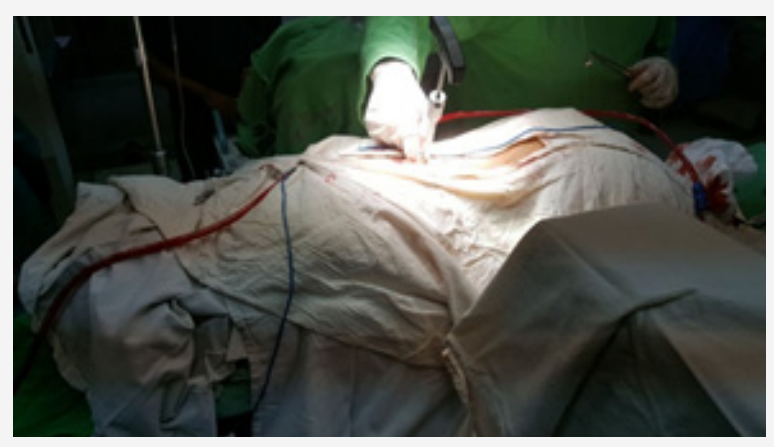

Figure 1: Patient positioning similar to conventional open approach with adequate padding at areas of contact (elbows and knees) to avoid pressure sores.

Surgical approach: Posterior approach was used.

Type of the construct: Top loading pedicle screws system.

Incision: Identification of surgical site by $\mathrm{C}$ arm and do marker. Two small a posterior midline incision at the target segment at the level of target pedicles determined by $\mathrm{C}$-arm. The length of the incision is about $2 \mathrm{~cm}$, one level above and below the fractured vertebra is exposed (Figure 2).

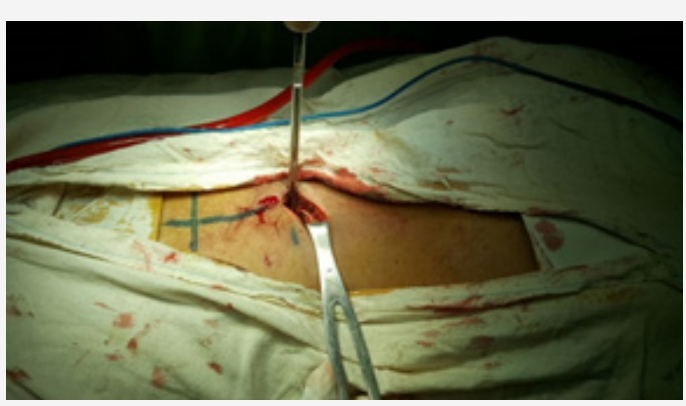

Figure 2: One level above and below the fractured vertebra was incised and the length of the incision was about $2 \mathrm{~cm}$. 
The standard posterior approach to the lumbar spine is made through a longitudinal incision. The thoracolumbar fascia is incised lateral to the supraspinous ligament, and the paraspinal muscles are raised laterally off the spinous processes, laminae, and facet joints with a Cobb elevator and a gauze swab. To expose the transverse processes for a Posterolateral fusion, the dissection continues down the lateral side of the superior facet and onto the transverse process. [8] During exposure, care is taken not to injure the facet joint capsule if a non-fusion technique is planned. With the help of mini-retractor (Figure 3).
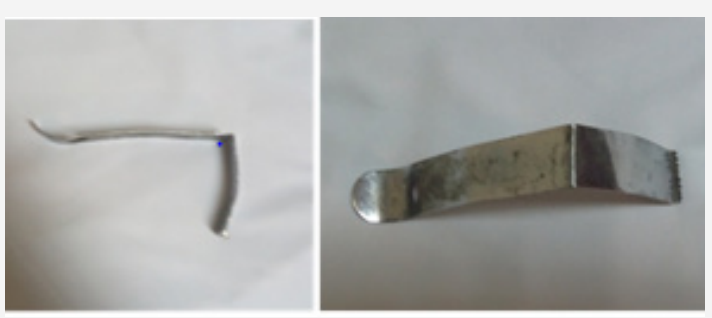

Figure 3: Mini-Retractor.

The entry point is now clearly exposed. The screw side Trajectory is identified and the screw insertion using free hand technique with $\mathrm{C}$ arm control under direct vision using $\mathrm{AO}$ technique. Screw placement was checked electro physiologically with direct stimulation of the pedicle probe or screw producing an electromyogram (EMG) response peripherally; if this response occurs below the threshold expected for intact cortical bone, the screws may be redirected or removed [9].

After application of the 4 screws Figure 4, a contour rod is pass Figure 5 sub muscular with minimal manipulation, essentially no muscle dissection (Figure 4\&5).

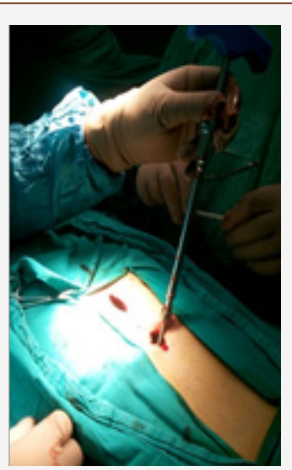

Figure 4: Insertion of the 4 pedicular screws.

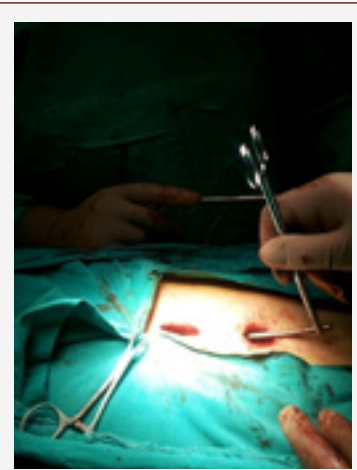

Figure 5: A contour rod is pass sub masculare.
Reduction and indirect decompression: Usually partial spontaneous reduction was achieved when the patient was turned to the prone position on a special frame that restored the normal dorsal kyphotic and lumbar lordotic curves of the spine. The reduction was checked by the image intensifier. Reduction and indirect decompression were done as follows: Correction of Kyphosis and recreation of normal lordosis were done by contouring the rod. Correction was achieved using the principle of 3 points fixation. The cephalic and caudal ends of the rods constitute 2 points of fixation and the third was the apex of the deformity that was ventrally displaced by tightening of the anchors. Pedicle screw fixation secured all 3 columns and provided excellent fixation.

Application of distraction by distractor and distraction was done until correction of vertebral height if possible guided by $\mathrm{C}$ arm control. The correction maintained by tightening screws head nuts (Figure 6\&7).

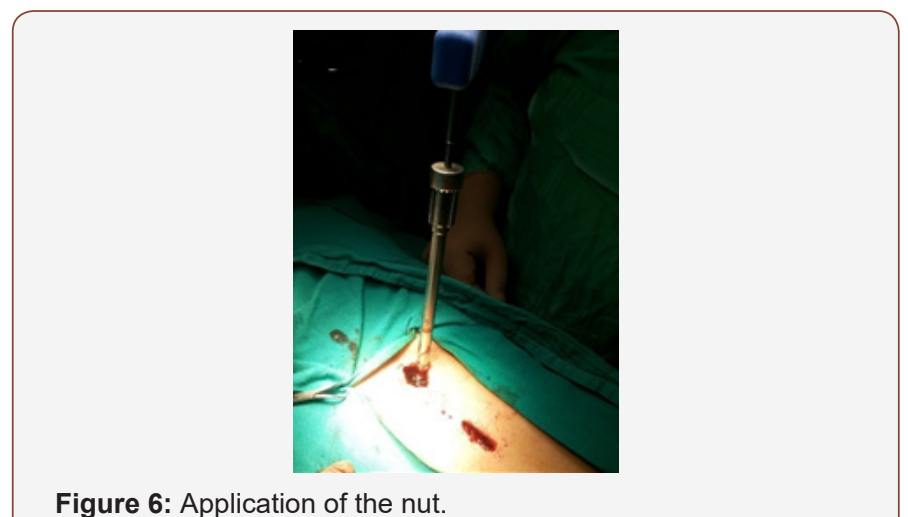

Figure 6: Application of the nut.

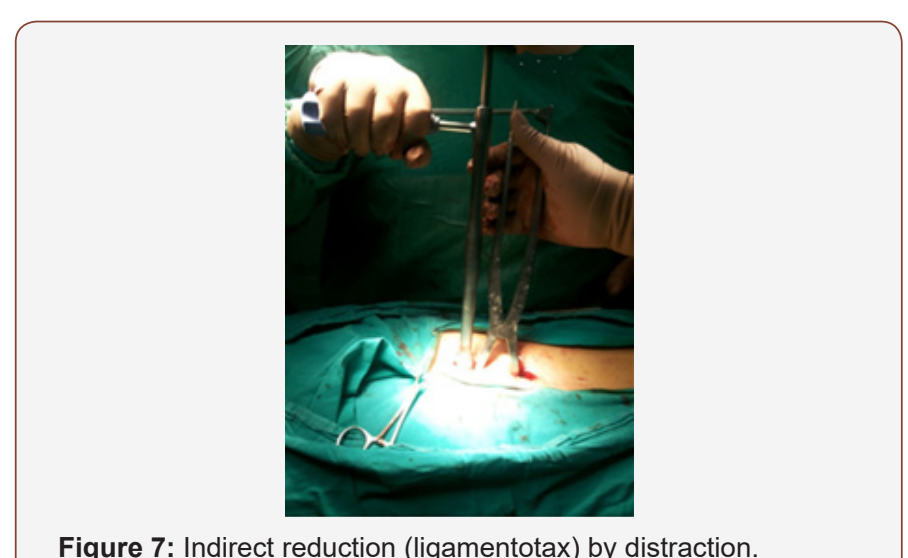

Figure 7: Indirect reduction (ligamentotax) by distraction.

The wound is then closed in layers muscles, fascia, subcutaneous layer and skin using absorbable sutures. In most of cases the wound did no need a drain.

\section{Postoperative care}

- $\quad$ All patients were neurologically tested before leaving the operating room. Patients were kept flat for a period of 24 hours after surgery with close observation to vital signs and drain if applied. Post-operative antibiotics were continued for 2 days.

- $\quad$ Post-operative X- ray and multi slice CT scans were performed to assess the screw position and then repeated after 6 months and may be repeated again till assurance of bone healing. Routine clinical and check-up was done every month 
postoperatively for first three months, then every three months afterwards (Figure $8 \&$ 9).

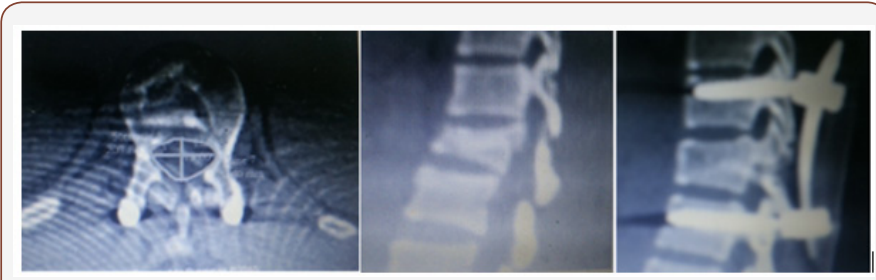

Figure 8: A. Post-operative CT diameter of spinal canal, B. Preoperative Kyphosis evaluation by CT and C. post-operative Kyphosis evaluation.

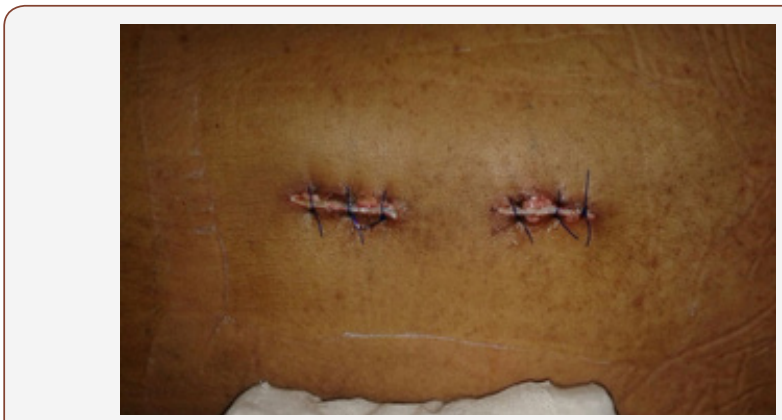

Figure 9: Structures at the end of surgery.

The study parameters included operative time, blood loss, postoperative drainage, postoperative hospital stays, X-ray exposure time, kyphotic angle, visual analog scale (VAS) scores, postoperative complications, the accuracy rate of screws placement of pedicle screws using classified of Bride Upendra of thel All India Institute of Medical Sciences (AIIMS) group [10]. Also, cosmetic appearance Figure 9. Clinical outcome was classified according to modified Mac nab criteria [11].

\section{Result}

All operations were performed using mini access technique apart from two cases. The operative time (minutes) $65.0 \pm 23.2$ with the longer operative times occurring early in the learning curve. The average blood loss is $94 \mathrm{ml}$ ranged from $70 \mathrm{ml}$ to 100 . Fluoroscopy time5.2 \pm 2 (seconds). 12 cases with postoperative drainage the Postoperative drainage $(\mathrm{mL})$ was $51.9 \pm 37.3$, all the patients were discharged on postoperative Day 1 or 2 apart from 7 patients were discharged on postoperative Day 5 and 9 days due to associated injuries with mean Postoperative hospital stay (days) $3.1 \pm 1.8$ days.

The follow-up period ranged from 6 to 18 months postoperative with an average of 10 months. All patients improved clinically, and outcome was classified using the modified Mac nab criteria Table 1. Total of 128 screws were applied of which 3 screws were mal positioned (2.3\%). The accuracy rate was $97.7 \%$. Open conversion surgery was done in two cases (6.25\%). The Accuracy rate of screws placement of pedicle screws using classification of Bride Upendra of the All India Institute of Medical Sciences Type I: position acceptable in 108 screws. In 16 screws were classified as Type II: placement unacceptable those that do not correspond to Type I and that do not show signs of neurovascular compromise. In 3 screws were classified as Type III: screws that are causing neurovascular injury) all cases causing radicular pain). There was no case of pull out of screws.
Were considered excellent in sixteen patients, good in fourteen patients, and poor in two patients. Figure 6 Although these latter two patients fared well clinically, they required reoperation for hardware revision due to mal insertion of three screws. Of the seventeen patients who had been followed for longer than 6 months, all were judged to have solid union. All the patients were satisfied from cosmetic appearance of the scar. Clinical outcome was classified according to modified Mac nab criteria [11] (Table 1 \& Figure 10).

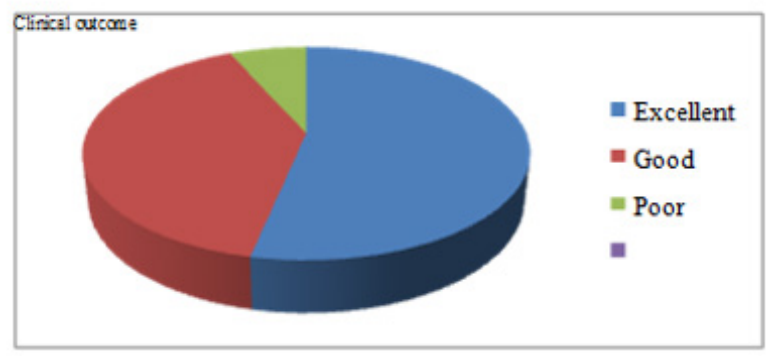

Figure 10: Results.

Table 1: Modified Mac Nab criteria used to grade overall Clinical outcome after spinal surgery.

\begin{tabular}{|c|c|}
\hline Excellent & $\begin{array}{r}\text { No pain, no restriction of mobility, Return to normal } \\
\text { work and level of activity }\end{array}$ \\
\hline Good & $\begin{array}{r}\text { Occasional non-radicular pain, Relief of presenting } \\
\text { symptoms, Able to return to modified work }\end{array}$ \\
\hline Fair & $\begin{array}{r}\text { Some improved functional capacity, Still handicapped } \\
\text { and/or unemployed }\end{array}$ \\
\hline Poor & $\begin{array}{r}\text { Continued objective symptoms of root involvement, } \\
\text { Additional operative intervention needed at index } \\
\text { level irrespective of length of postoperative follow-up. }\end{array}$ \\
\hline
\end{tabular}

Table 2: Mean Vertebral Height Index (VHI).

\begin{tabular}{|c|c|c|c|}
\hline Level Number & Pre-0p & Post-Op & Follow-Up \\
\hline D 12 8 p & 58 & 86 & 84 \\
\hline L1 17 p & 66 & 89 & 85 \\
\hline L2 4 p & 62 & 87 & 85 \\
\hline L3 3 p & 75 & 91 & 88 \\
\hline
\end{tabular}

L=lumbar, $\mathrm{D}=$ dorsal, $\mathrm{P}=$ patient

Table 3: Mean canal area in $\mathrm{mm}^{2}$.

\begin{tabular}{|c|c|c|c|}
\hline Level Number & Pre-0p & Post-0p & Last follow up \\
\hline D 12 8 p & 182.34 & 211.33 & 207.51 \\
\hline L1 17 p & 177.42 & 207.94 & 205.3 \\
\hline L2 4 p & 168.2 & 181.73 & 178.29 \\
\hline L3 3 p & 189.3 & 204.22 & 202.75 \\
\hline
\end{tabular}

$\mathrm{L}=$ lumbar, $\mathrm{D}=$ dorsal, $\mathrm{P}=$ patient

Preoperative pain was high in all patients, with the mean visual analogue scale value of 7.9/10 (range 6-10). It decreased to 2.2 at time of discharge (range 0-7) apart from the two cases which need revision and the pain was dramatically improved and to 0.7 at the last follow-up (range 0-2). There were improvements in the vertebral Kyphosis, vertebral height index and canal area in immediate postoperative CT scans which persisted on last follow-up (varying from 6 to 12 months) Table 2, there were an improvement in the diameter 
of the canal area comparing preoperative to post-operative (Table 2\&3).

\section{Cases presentation}

Case male patient 40 years old, fallen from height has L1 fracture

The following (Figure 11)

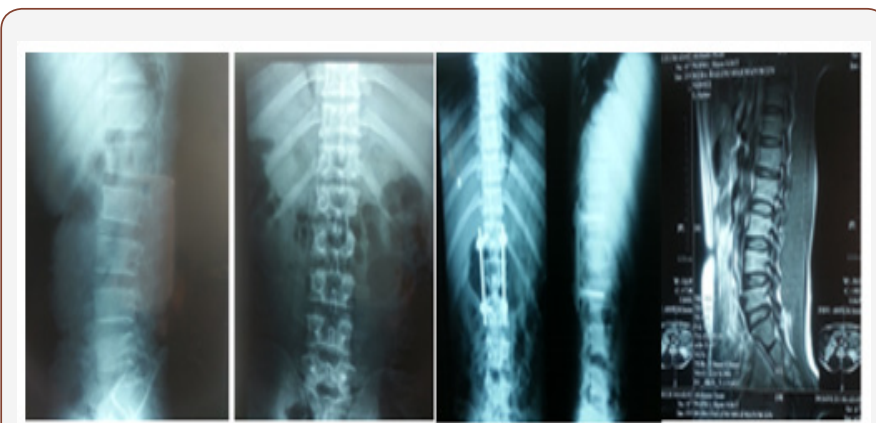

Figure 11: a) AP lateral Lat. View, b) AP MRI, C) post-operative AP d) AP Lat. View.

\section{Discussion}

The treatment of traumatic fractures of the thoracic and lumbar spine remains controversial. There is insufficient evidence in the literature to choose between the various surgical options. Five surgical subgroups were recognized: posterior short-segment (PS), posterior long-segment (PL), reports on both posterior shortand long-segment (PSL), anterior (A), and anterior combined with posterior (AP) techniques [12]. Patients with thoracolumbar spine fracture without neurologic deficit should be treated by short-segment posterior stabilization [13,14]. Single-segment thoracolumbar fractures without neurologic injury treated by pedicle screw fixation. Posterior short-segment (PS), surgical technique can be either through posterior midline incision or paraspinal posterior open approaches or Percutaneous [15]. Wiltse introduce a novel mini-open pedicle screw fixation technique via Wiltse approach [16].

Each method had advantage and disadvantages. It is known that traditional posterior open approaches to spine surgery lead to increased paraspinal muscle injury following denervation, ischemia secondary to prolonged retraction and detachment of musculotendinous junction. Denervation and ischemia can result from direct injury to dorsal roots and vasculature in extensive surgical exposure and also occurs due to increased intramuscular edema and resultant focal compartment syndrome secondary to prolonged strong retraction [17]. Minimally invasive spine surgery (MISS) is an alternative to traditional open surgical procedures performed to treat different spinal disorders, Spine surgery performed minimally invasively offers many potential benefits, such as small incisions, less cutting through soft tissues (eg. ligaments, muscles), outpatient options, less post-operative pain, and faster recovery.

In 2016 we introduce the novel Minimally Invasive ((Mini access)) open Spine Osteosynthesis (MISO) Technique [18]. Potential advantages compared with "open" surgery, may result in Smaller incisions and scars, Minimal soft-tissue destruction and scarring, less surgical blood loss, no special instrument or screws, shorter hospital stay, less postoperative painless need for postoperative pain medicine, faster returns to work and daily activities.

The procedure involves minimal muscle retraction and minimal stripping of muscles. The pedicles are addressed by usual steps and no needs for specialized instruments. The rod is delivered into the screw head deep in the tissue and tightened in place with set screws. This surgery approach allows to be performed with traditional instruments; On the other hand, the amount of radiation that the patient and surgeon are exposed is less than with minimally invasive spine fusion systems than the specific system being used. Incisions: it leaves 2 small scars of approximately less than 2 centimeters, one scar to upper vertebra and one to lower fixed vertebra in the midline. From point of recovery the patient can leave hospital next day, Operative Time: The amount of surgeon experience is by far the most important factor in operative times rather than the specific spine fusion surgery system used. In general, posterior instrumentation in general most of surgeon can do it as they trained in Posterolateral fusions.

The need of blood transfusion is decreased and none of our patients required it. Surgeon learning curve: Pedicle targeting is all spinal surgeons are family with it, so a steep learning curve for the surgeon to build the necessary skills and experience are minimal. Compared to the traditional open spine surgery, mini access techniques require smaller incisions and decrease approachrelated morbidity.

Schwender et al. [19] described their technique of mini PLIF. The mean blood loss of $140 \mathrm{ml}$ and mean hospital stay of 1.9 days in their series is comparable to that of $94 \mathrm{ml}$ and 3.1 days respectively in our study. The excellent 16 and good 14 clinical outcomes in our study of $93.75 \%$ near to that of $89.4 \%$ of Schwender [19]. The accuracy rate of pedicle screw placement in open was $96.6 \%$., mini-open using Wiltse approach; the accuracy rate was $95.7 \%$. Comparing to our study the accuracy rate was $97.7 \%$. The biggest current limitation of the mini access approach is that it is not suitable for cases need posterior laminectomy, decompression and Posterolateral fusion.

\section{Conclusion}

Although this study is limited by its retrospective nature and small sample size, the results demonstrate mini access spine surgery is cost effective minimal soft tissue dissection with short hospital stay. The authors believe that the data support its use and that this technique may be applicable for cases not needs spinal decompression, Mini access spinal technologies enable surgeons to achieve the same surgical objectives as with a traditional, open procedure.

\section{Acknowledgement}

None.

\section{Conflict of Interest}

No conflict of interest. 


\section{References}

1. Roy Camille R, Saillant G, Mazel C (1986) Plating of thoracic, thoracolumbar and lumbar injuries with pedicle screw plates. Orthop Clin North Am 17(1): 147-159.

2. Gaines RW (2000) The use of pedicle-screw internal fixation for the operative treatment of spinal disorders. J Bone Joint Surg Am 82-A (10): 1458-1476.

3. Yuan HA, Garfin SR, Dickman CA, Mardjetko Steven M (1994) A historical cohort study of pedicle screw fixation in thoracic, lumbar, and sacral spine fusions. Spine j 19(20): 2279S-2296S.

4. Dick W, Kluger P, Magerl F, Woersdorfer O, Zach G (1985) A new device for internal fixation of thoracolumbar and lumbar spine fractures: The 'fixateur interne'. Paraplegia 23(4): 225-232.

5. Vaccaro AR, Zeiller SC, Hulbert RJ, Anderson PA, Harris M, et al. (2005) A new classification of thoracolumbar injuries: the importance of injury morphology, the integrity of the posterior ligamentous complex, and neurologic status. Spine (Phila Pa 1976) 30(20): 2325-2333.

6. Lowery GL, Kulkarni SS (2000) Posterior Percutaneous spine instrumentation. Eur Spine J 9(1): S126-S130.

7. Joon Y Lee, Alexander R Vaccaro, Moe R Lim, FC Öner, R John Hulbert (2005) Thoracolumbar injury classification and severity score: a new paradigm for the treatment of thoracolumbar spine trauma. J Orthop Sci 10(6): 671-675.

8. Weinstein JN (1994) Surgical approaches to the lumbar spine. In: Weinstein SL (Ed.) The pediatric spine: principles and practice. Raven, New York, USA, pp. 1369-1380.

9. DH Clements, DE Morledge, WH Martin, RR Betz (1996) Evoked and spontaneous electromyography to evaluate lumbosacral pedicle screw placement. Spine (Phila Pa 1976) 21(5): 600-604.

10. Upendra BN, Meena D, Chowdhury B, Ahmad A, Jayaswal A (2008) Outcome-based classification for assessment of thoracic pedicular screw placement. Spine (Phila Pa 1976) 33(4): 384-390.
11. Macnab I (1971) Negative disc exploration. An analysis of the causes of nerve-root involvement in sixty-eight patients. J Bone Joint Surg Am 53(5): 891-903.

12. Verlaan JJ, Diekerhof $\mathrm{CH}$, Buskens E, van der Tweel I, Verbout AJ, et al. (2004) Surgical Treatment of Traumatic Fractures of the Thoracic and Lumbar Spine: A Systematic Review of the Literature on Techniques, Complications, and Outcome. Spine (Phila Pa 1976) 29 (7): 803-814.

13. Siebenga J, Leferink VJ, Segers MJ, Elzinga MJ, Bakker FC, et al. (2006) Treatment of Traumatic Thoracolumbar Spine Fractures: A Multicenter Prospective Randomized Study of Operative Versus Nonsurgical Treatment. Spine (Phila Pa 1976) 31(25): 2881-2890.

14. Denis F, Armstrong GW, Seals K, Matta L (1984) Acute thoracolumbar burst fractures in the absence of neurologic deficit. A comparison between operative and nonoperative treatment. Clin Orthop Relat Res 189: 142-149.

15. Xiang Yao Sun, Xi-Nuo Zhang, Yong Hai (2017) Percutaneous versus traditional and paraspinal posterior open approaches for treatment of thoracolumbar fractures without neurologic deficit: a meta-analysis. Eur Spine J 26(5): 1418-1431.

16. Wiltse LL, Bateman JG, Hutchinson RH, Nelson WE (1968) The paraspinal sacrospinalis-splitting approach to the lumbar spine. J Bone Joint Surg Am 50(5): 919-926.

17. Rampersaud YR, Foley KT, Shen AC, Williams S, Solomito M (2000) Radiation exposure to the spine surgeon during fluoroscopically assisted pedicle screw insertion. Spine (Phila Pa 1976) 25(20): 2637-2645.

18. Kornah BA, safwat HM, Abdel Aal MA (2016) Minimally Invasive Spine Osteosynthesis (MISO) Technique for Fractures Spine: A Case Series 14 Cases. J Trauma Treat 5: 336.

19. Schwender JD, Holly LT, Rouben DP, Foley KT (2005) Minimally invasive transforaminal lumbar interbody fusion (TLIF); technical feasibility and initial results. J Spinal Disord Tech 18: S1-6. 